\title{
Engineering Notes
}

\section{Fast Large-Eddy Simulation of Low Reynolds Number Flows over a NACA0025}

\author{
Tao Xu* and Pierre Sullivan \pm \\ University of Toronto, Toronto, Ontario M5S 3G8, Canada \\ and \\ Marius Paraschivoiu声 \\ Concordia University, Montreal, Quebec H3G 1M8, Canada
}

DOI: $\underline{10.2514 / 1.44251}$

\section{Introduction}

B LADES of small vertical-axis wind turbines operate at low Reynolds numbers and undergo continuous dynamic stall [1]. To improve the design of these blades, flow features such as separation and turbulent transition need to be captured accurately, which is still a challenge for most of the computational fluid dynamics codes. In a first attempt to simulate these flows, a code is developed and tested on a static airfoil at a low Reynolds number.

When the chord Reynolds number $R e_{c}$ decreased below 500,000 the airfoil performance deteriorated by losing lift and increasing drag [2]. A free shear layer forms as the laminar boundary layer separates in an adverse pressure gradient forming a laminar separation bubble. This separated laminar boundary-layer transition to turbulence over an airfoil is a complex problem that is not completely understood. In particular, the information obtained so far is inadequate for clarifying the entire transition process, large-scale structures, and the instability mechanisms.

Recently, an extensive experimental study of boundary-layer development for a NACA0025 airfoil at low Reynolds number was experimentally conducted [3]. Flow velocity data were obtained with hot-wire anemometry. Wind-tunnel experiments were carried out for a range of Reynolds numbers from $10^{5}$ to $10^{6}$ and three angles of attack $\left(5,10\right.$, and $\left.15^{\circ}\right)$. Two boundary-layer regimes were identified: 1) boundary-layer separation without reattachment and 2) separation bubble formation. Their results showed that transition to turbulence, which occurs because of the amplification of disturbances in the separated shear layer, plays a key role in boundary-layer reattachment. Their results also suggest that coherent structures form in the separated flow region and the formation of the roll-up vortices in the separated shear layer is linked to inviscid spatial growth of disturbances and is attributed to the Kelvin-Hemholtz instability. The final stage of transition is associated with the growth of a subharmonic component in the velocity spectrum, which can be attributed to the merging of the roll-up vortices.

Presented as Paper 4199 at the 18th AIAA Computational Fluid Dynamics Conference, Miami, FL, 25-28 June 2007; received 9 March 2009; accepted for publication 24 September 2009. Copyright (C) 2009 by the American Institute of Aeronautics and Astronautics, Inc. All rights reserved. Copies of this paper may be made for personal or internal use, on condition that the copier pay the $\$ 10.00$ per-copy fee to the Copyright Clearance Center, Inc., 222 Rosewood Drive, Danvers, MA 01923; include the code 0021-8669/10 and $\$ 10.00$ in correspondence with the CCC.

*Department of Mechanical \& Industrial Engineering; taoxu@ mecheng1. uwaterloo.ca.

Department of Mechanical \& Industrial Engineering; sullivan@mie. utoronto.ca.

†Department of Mechanical \& Industrial Engineering; paraschi@me. concordia.ca.
Given that the airfoil boundary layer at low Reynolds number is inherently unsteady and features important large-scale motions, this flow is not well predicted by Reynolds-averaged Navier-Stokes models. Instead a time-dependent simulation, such as a direct numerical simulation or large-eddy simulation (LES), is required for more accurate results.

Large-eddy simulation is particularly suitable to investigate the generation and evolution of coherent structures in turbulent flows. It is ideal for low Reynolds number flows and explicit computation of coherent structures and has only limited sensitivity to modeling assumptions so that results are feasible for this practical problem.

Flow past an experimentally studies NACA0025 airfoil is a challenging case for LES because of the different flow regimes around the airfoil, including the laminar boundary layer, laminar separation bubble, transition region, turbulent boundary layer, separation point, and separation region as well as wake region. In LES, it is important to resolve all important (large-scale) flow structures and model the remaining small-scale turbulence. Ideally, the goal would be to resolve the following flow regions: the laminar boundary layer with a sufficient amount of nodes (in the streamwise and wall-normal directions), the recirculation region (at the transition region and at the trailing edge), and the turbulent boundary layer. For blade design, it is also important to solve the flow in a short computational time. The code developed herein continues previous efforts to make LES applicable to complex flow simulations. The previous developed flow simulation methodology [4, 5$]$ also aims at obtaining accurate results on fewer grid nodes than standard LES.

Although LES is promising, the drawback of LES is the large memory required if all the above mentioned regions are resolved. To control the size of the problem, an instantaneous wall function [6] is used, allowing the first nodes near the airfoil surface to be placed in the logarithm layer instead of in the sublayer.

To further reduce computational cost, a pressure and velocity decoupled system is used. The consistent splitting method [7] evaluates the pressure by testing the momentum equation against gradients. The resulting Poisson equation for the pressure correction term avoids artificial boundary conditions. This scheme is free of splitting errors and the pressure error is smooth. Nonconforming finite elements with a lower requirement for degrees of freedom are chosen when compared to other conforming finite elements (e.g., the Taylor Hood element). This is a significant savings in memory and was suggested by [8] and implemented in FEATFLOW.

Highly nonuniform meshes satisfy the resolution requirements of LES in the boundary layer. A traditional solver cannot damp the high frequencies generated by nonuniform meshes effectively, and a multigrid method is often needed. But the filtering operations on all the multigrid scales have to be separated from the traditional multigrid procedure to prevent errors between the different filtering scales. To get good LES results, a filtering operation is developed in the present work and discussed in Sec. II.

In LES, the assumption of constant viscosity can not be made because of the eddy viscosity model used. This leads to a full coupling of the velocity components if an implicit treatment is applied for the viscous term. Two approaches have been traditionally used. One is the full coupled iterative method that can be solved iteratively but is expensive. The other is to treat the viscous term explicitly at the expense of reductions in the admissible time-step size due to stability restrictions.

A third variable-viscosity formulation [9] can be used to discretize the variable-viscosity term. However, stability is not ensured because the variable-viscosity term is included on the right-hand side; in addition, this approach is computationally expensive.

To simplify the above approach, a new scheme is adopted, where the variable viscosity is assumed piecewise constant in each element. 
Because no extra explicit terms appear on the right-hand side, stability is ensured; furthermore, the coupled equations are avoided.

To solve this problem for multigrid method and ensure that all boundary nodes are on the actual domain boundary, functions [8] are used to define the boundaries in advance. The geometry is respected during the refinement procedure with a parametric function for the boundaries.

This paper describes the LES equations and the finite element formulation used. A description of the extension of the dynamic subgrid model to nonconforming element simulations is presented. A new piecewise-constant approach to implement LES is discussed. A consistent splitting method to solve the resulting matrix system is introduced. Results of simulation are provided to illustrate the accuracy of the approach.

\section{Numerical Formulation}

The filtered Navier-Stokes equations are

$$
\begin{gathered}
\frac{\partial \bar{u}_{i}}{\partial t}+\frac{\partial}{\partial x_{j}}\left(\bar{u}_{i} \bar{u}_{j}\right)=-\frac{\partial \bar{p}}{\partial x_{i}}+\frac{\partial}{\partial x_{j}}\left[v\left(\frac{\partial \bar{u}_{i}}{\partial x_{j}}+\frac{\partial \bar{u}_{j}}{\partial x_{i}}\right)\right]-\frac{\partial \tau_{i j}}{\partial x_{j}} \\
\frac{\partial \bar{u}_{i}}{\partial x_{i}}=0
\end{gathered}
$$

where

$$
\tau_{i j}=\overline{u_{i} u_{j}}-\bar{u}_{i} \bar{u}_{j}
$$

is the subgrid-scale stress tensor, which is modeled as

$$
\tau_{i j}-\frac{1}{3} \delta_{i j} \tau_{k k}=-2 v_{\mathrm{sgs}} \bar{S}_{i j}
$$

where $\bar{S}_{i j}$ is the strain rate tensor. The Smagorinsky model is used to describe $v_{\text {sgs }}$

$$
v_{\mathrm{sgs}}=\left(C_{s} \Delta\right)^{2}|\bar{S}|
$$

where $|\bar{S}|=\sqrt{2 \bar{S}_{i j} \bar{S}_{i j}}$ is the norm of the large-scale strain rate tensor, and $C_{s}$ is the Smagorinsky constant, which can be determined dynamically using the following equations:

$$
C_{s} \Delta^{2}=\frac{1}{2} \frac{\left\langle M_{i j} L_{i j}\right\rangle}{\left\langle M_{k l} M_{k l}\right\rangle}
$$

where

$$
\begin{gathered}
M_{i j}=\left\|\widehat{\bar{S} \| \bar{S}_{i j}}-\left(\frac{\hat{\bar{\Delta}}}{\bar{\Delta}}\right)^{2}\right\| \hat{\bar{S}} \| \widehat{\overline{S_{i j}}} \\
L_{i j}=\widehat{\overline{\bar{u}}_{i} \bar{u}_{j}}-\widehat{\widehat{\bar{u}}_{i}} \widehat{\bar{u}}_{j}
\end{gathered}
$$

The hat symbol indicates explicit application of a secondary filter with kernel $\hat{G}$ and width $\hat{\Delta}$, as a test filter. The brackets denote averaging over spatially homogeneous directions which was done to avoid large negative values of $C_{s}$. In this work, artificial bounds are used for $C_{s}$. The upper limit is 0.27 and the lower limit is -0.01 to address the backscatter in the transitional flows while retaining numerical stability.

Nonconforming finite elements based on rotated multilinear shape functions [8] were introduced as a class of simple compatible elements for the Stokes problem, and the pressure is piecewise constant. More generally, the recent development of efficient solution methods for nonconforming finite element systems are available because of the stable discretization for ill-conditioned problems.

This element pair has several important features. It admits simple upwind strategies which may lead to matrices with certain $M$-matrix properties. Efficient multigrid solvers are available, which work satisfactorily over the whole range of relevant Reynolds numbers, $1<\operatorname{Re}<10^{5}$. The Reynolds number is based on chord length.

In addition, less memory is required for the element pair [8] used in this work. It has a maximum band width of 11 in three dimensions, as compared to the corresponding number for a conforming triquadratic function of at least 125. Substituting the basis functions into the variational form of the Navier-Stokes equation gives the following semidiscrete equations:

$$
\begin{gathered}
\mathbf{M} \frac{\partial u_{i}^{h}}{\partial t}+\mathbf{C}\left(\mathbf{u}^{\mathbf{h}}\right) u_{i}^{h}+\sum_{k_{e}=1}^{N^{\mathrm{el}}} v_{t}^{k_{e}} \mathbf{A}^{h, k_{e}} u_{i}^{h, k_{e}}-\mathbf{D}_{i}^{T} p^{h}=0 \\
i=1,2,3 \\
\mathbf{D}_{\mathbf{i}} \overline{\mathbf{u}}_{\mathbf{i}}^{\mathbf{h}}=\mathbf{0}
\end{gathered}
$$

where $\mathbf{M}$ is the resulting mass matrix, $\mathbf{C}\left(\mathbf{u}^{\mathbf{h}}\right)$ is the matrix for the convection term, $\mathbf{A}^{h, k_{e}}$ is stiffness matrix, and $\mathbf{D}$ is the gradient matrix.

The term

$$
\sum_{k_{e}=1}^{N^{\mathrm{el}}} v_{t}^{k_{e}} \mathbf{A}^{h, k_{e}} u_{i}^{h, k_{e}}
$$

is new and is adopted to simplify the discretization of variable viscosity $v_{t} . v_{t}$ is the total viscosity defined as the sum of the molecular viscosity and eddy viscosity. Because eddy viscosity varies in space, $v_{t}$ is not a constant. In this work, $v_{t}$ is assumed to be piecewise constant in each element, which greatly simplifies the scheme proposed by [9].

However, the $v_{\text {sgs }}$ calculated from the Smagorinsky model is related to the velocity gradients of large eddies, which are at different locations of the elements. In this work, a modified Smagorinsky model was implemented by finite element method to calculate the elementwise average of the $v_{\text {sgs }}$ instead,

$$
v_{\mathrm{sgs}}^{k_{e}}=\left(C_{s} \Delta\right)^{2} \frac{\int_{\Omega^{e}}|S|^{k_{e}} \mathrm{~d} \Omega}{\int_{\Omega^{e}} \mathrm{~d} \Omega}
$$

The advantages of this new approach are as follows:

1) For nonconforming elements, the nodal values and their derivatives are discontinuous. To calculate the derivatives, it is possible only to calculate the element-based derivatives first, then to project them onto the nodes. This leads to solving nodal-based derivatives across the whole domain. By calculating the element average of the derivative instead, the calculations become computationally inexpensive as well as easily implemented.

2) The $v_{\text {sgs }}$ is smooth and has no high-frequency component.

3) The elemental $v_{\mathrm{sgs}}^{k_{e}}$ is piecewise constant after the above treatment.

\section{A. Temporal Discretization}

The strategy for temporal discretization is a second-orderaccurate, implicit backward differentiation BD2 scheme.

Equation (16) can be fully discretized as

$$
\begin{gathered}
\mathbf{M}\left(\zeta_{\mathbf{1}} \overline{\mathbf{u}}_{\mathbf{i}}^{\mathbf{n}+\mathbf{1}}+\zeta_{\mathbf{2}} \overline{\mathbf{u}}_{\mathbf{i}}^{\mathbf{n}}+\zeta_{\mathbf{3}} \overline{\mathbf{u}}_{\mathbf{i}}^{\mathbf{n}-\mathbf{1}}\right)+\mathbf{C}\left(\mathbf{u}^{\mathbf{n}+\mathbf{1}, \mathbf{h}}\right) \mathbf{u}_{\mathbf{i}}^{\mathbf{n}+\mathbf{1}, \mathbf{h}} \\
+\sum_{k_{e}=1}^{N^{\mathrm{el}}} \Delta v_{t}^{k_{e}} \mathbf{A}^{h, k_{e}} u_{i}^{n+1, h, k_{e}}-\mathbf{D}_{i}^{T} p^{n+1, h}=0 \\
i=1,2,3
\end{gathered}
$$

The standard coefficients for the $B D 2$ scheme are given by

$$
\zeta_{1}=\frac{3}{2 \Delta t}, \quad \zeta_{2}=-\frac{2}{\Delta t}, \quad \zeta_{3}=\frac{1}{2 \Delta t}
$$

An uping approach was used to stabilized the convection matrix, in which $\tilde{n}_{h}\left(u_{h}, v_{h}, w_{h}\right)$ replaces $\mathbf{C}\left(\mathbf{u}^{n+1, h}\right)$. 


$$
\begin{aligned}
& \tilde{n}_{h}\left(u_{h}, v_{h}, w_{h}\right):=\sum_{l} \sum_{k \in \Lambda_{l}} \oint_{\Gamma_{l k}} u_{h} \cdot n_{l k} \mathrm{~d} \gamma\left(1-\lambda_{l k}\left(u_{h}\right)\right)\left(v_{h}\left(m_{k}\right)\right. \\
& \left.\quad-v_{h}\left(m_{l}\right)\right) w_{h}\left(m_{l}\right)
\end{aligned}
$$

where

$$
\lambda_{l k}(x):=\left\{\begin{array}{ll}
\frac{0.5+\alpha x}{1+\alpha x} & \text { if } x \geq 0 \\
\frac{1}{2(1-\alpha x)} & \text { otherwise }
\end{array}\right\}
$$

and $\alpha$ is a chosen damping parameter.

For the above upwind scheme, it can be shown in one dimension [8] that the resulting discretization is of second-order accuracy. The corresponding stiffness matrix $S_{h}$ for velocity components can be an $M$ matrix [8], which results in very nice linear algebraic properties, concerning convergence results for Jacobi and successive overrelaxation iterative methods. This is essential for the existing multigrid solution tools we use. The detailed discussion of Eq. (14) is found in [10].

The stability limit on the $\Delta t$ is determined by consistent splitting method discussed below.

\section{B. Consistent Splitting Method}

For computational efficiency, a splitting method is employed that decouples pressure from the system [7]. This algorithm is similar to Chorin's approach, which calculated the intermediate velocities. The next step of the algorithm takes care of the incompressibility constraint; the projection step. It is a Poisson equation supplemented with Neumann boundary conditions.

$$
\mathbf{E} \psi^{n+1}=\frac{1}{\Delta t} \nabla \cdot u^{n+1}, \quad \partial_{n} \psi_{\Gamma}^{n+1}=0
$$

where $\Gamma$ is the boundary and the Poisson operator $\mathbf{E}$ is defined as

$$
\mathbf{E}=\mathbf{D}_{1} \mathbf{M}^{-1} \mathbf{D}_{1}^{T}+\mathbf{D}_{2} \mathbf{M}^{-1} \mathbf{D}_{2}^{T}+\mathbf{D}_{3} \mathbf{M}^{-1} \mathbf{D}_{3}^{T}
$$

To simplify the solving of Eq. (16), a lumped mass matrix is used instead of inverting the mass matrix.

The elemental lumped mass matrix is defined as

$$
M^{k_{e}}=\frac{M_{i i}^{k_{e}}}{\operatorname{Trace}\left(M^{K_{e}}\right)} \operatorname{vol}^{K_{e}}
$$

A pressure correction term is used as follows:

$$
p^{n+1}=\psi^{n+1}+p_{*}^{n+1}-v \nabla \cdot u^{n+1}
$$

where

$$
p_{\star}^{n+1}=\left\{\begin{array}{c}
p^{0} \text { for } k=0 \\
p^{1}+2 \psi^{1} \text { for } k=1 \\
p^{2}+\frac{4}{3} \psi^{2}-\frac{1}{2} \psi^{1} \text { for } k=2 \\
p^{k}+\frac{4}{3} \psi^{k}-\frac{1}{3} \psi^{k-1} \text { for } k \geq 3
\end{array}\right\}
$$

$k=1$ is used in our implementation. This pressure update is better calculated by solving the following weak form problem: For all $q_{h} \in M_{h}$,

$$
\left(p_{h}^{n+1}, q_{h}\right)=\left(\psi_{h}^{n+1}+p_{h_{*}}^{n+1}-v \nabla \cdot u_{h}^{n+1}, q_{h}\right)
$$

This algorithm yields a smooth pressure field near the boundary, and the pressure approximation $p^{k+1}$ is no longer affected by an artificial
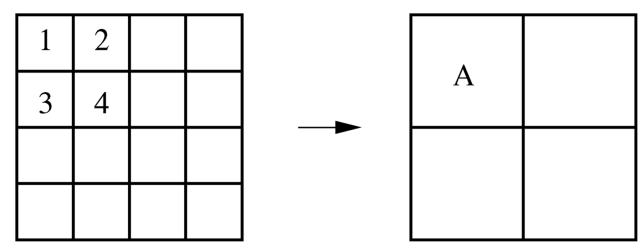

Fig. 1 Interpolation of eddy viscosity from fine mesh to coarse mesh.

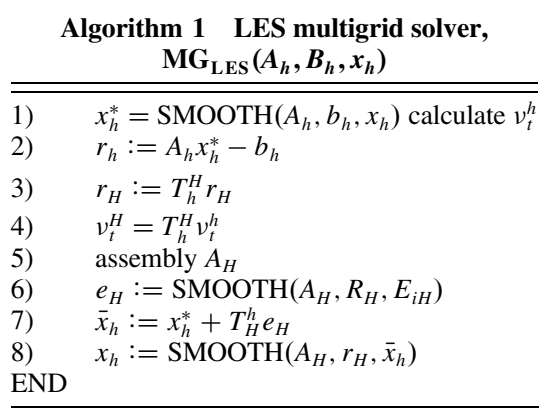

Neumann boundary condition. Details of the algorithms and a summary of the numerical approach are found in [11].

\section{Modified Multigrid Solver for LES}

An existing multigrid solver was chosen and modified to solve the algebraic system generated by large-eddy simulations.

As illustrated in Fig. 1, the eddy viscosity is first calculated based on the finest mesh. To obtain the eddy viscosity at the coarser scale (i.e., eddy viscosity for element A), the eddy viscosity of elements 1 to 4 of the finest mesh is used to calculate the corresponding eddy viscosity of element $A$. The new matrix is then assembled based on the eddy viscosity interpolated from the finest mesh. Thus, the twolevel scheme for LES is modified and presented below. Smooth is a smoothing step of the solution, where several iterations are performed using an iterative solver. Lines 2 and 3 are the restriction step, whereas line 7 is the prolongation step. An initial guess for $x_{h}$ is used on line 1 to start the process and is updated on line 8 at the end of the Algorithm 1 .

\section{Numerical Results}

The NACA0025 airfoil studied in [3] is used herein to validate the numerical approach. Because the aspect ratio is 2.93 , with a chord length $c$ of $0.3 \mathrm{~m}$, the flow at midspan can be considered twodimensional based on the experimental observation [3]. The computational domain is chosen to match the experiment [3] and extends $0.5 c$ upstream of the leading edge and $1 c$ downstream of the trailing edge. The spanwise width is $h=0.15 c$ in the experiment, we chose $h=1.1 c$ instead to enhance flow stability at the midspan. The airfoil is placed in a tunnel with a height of $1.2 \mathrm{~m}$.

The velocities are set to $\bar{u}=U_{\infty} \cos \alpha$ and $\bar{v}=U_{\infty} \sin \alpha$, where $U_{\infty}$ is the freestream velocity and $\alpha$ is the angle of attack. At the outflow boundary, an homogenous Neumann boundary condition is enforced and no-slip boundary conditions are used for the boundary of the airfoil and four tunnel walls.

A two-dimensional grid was initially generated by OMEGA then extruded to 3-D with 10 slices for the coarse mesh. The multigrid level increases in the code automatically refines the mesh. In addition, the LES grid places $10-15$ points inside the suction side boundary layer close to the trailing edge.

In this work, an approximate numerical method is used to estimate the friction velocity based on the turbulent boundary-layer scale at the first time step, the wall shear stress is

$$
\tau_{w}=\frac{0.0135 \mu^{1 / 7} \rho^{6 / 7} u_{\infty}^{13 / 7}}{L^{1 / 7}}
$$

Because

$$
\tau_{w}=\rho u_{\tau}^{2}
$$

where $u_{\tau}$ can be calculated. After the first time step, the wall function is used to calculate the local friction velocity.

Table 1 shows friction velocity for two different Reynolds numbers.

For this airfoil, separation takes place at the position of curvature change. At the separation point, the wall shear stress is zero, 
Table 1 Comparison of friction velocity

\begin{tabular}{lcc}
\hline \hline Reynolds number $R e_{c}$ & Velocity $u$ & Friction velocity $u_{\tau}$ \\
\hline $10^{5}$ & $5.0 \mathrm{~m} / \mathrm{s}$ & $0.252 \mathrm{~m} / \mathrm{s}$ \\
$1.5 \times 10^{5}$ & $7.5 \mathrm{~m} / \mathrm{s}$ & $0.367 \mathrm{~m} / \mathrm{s}$ \\
\hline \hline
\end{tabular}

Table 2 Comparison of near-wall resolution

\begin{tabular}{lcc}
\hline \hline Reynolds number $R e_{c}$ & Velocity $u$ & Near-wall resolution \\
\hline $10^{5}$ & $5.0 \mathrm{~m} / \mathrm{s}$ & $1.81 \times 10^{-3} \mathrm{~m}$ \\
$1.5 \times 10^{5}$ & $7.5 \mathrm{~m} / \mathrm{s}$ & $1.24 \times 10^{-3} \mathrm{~m}$ \\
\hline \hline
\end{tabular}

therefore, the above estimation of friction velocity can be regarded as the maximum friction velocity for the airfoil.

In LES, the distance from the wall to the first node $\left(y^{+}\right)$must be less than two to resolve the velocity gradient close to the wall. For $R e_{c}=10^{5}, \Delta y=2 \times v / u_{\tau}=1.2 \times 10^{-4}$; but for $R e_{c}=5 \times 10^{5}$, the $\Delta y$ increases to $2.5 \times 10^{-5}$. This requirement implies a computational mesh of roughly $10^{8}$ nodes. Such spatial resolution makes wall-resolved LES numerically very expensive. As one of the main objective of this work is to reduce the computational effort while ensuring a fair resolution of the vortical structure, a wall function in the logarithmic region $\left(y^{+} \geq 15\right)$ was employed.

The new instantaneous wall functions [12] assume that the first node is located in the logarithmic region away from the wall boundary $\left(y^{+} \geq 11.63\right)$. The instantaneous logarithmic law of wall was used. The logarithmic law can be used to solve the friction velocity $u_{\tau}$ and the wall shear stress is

$$
\frac{\tau_{\mathrm{wall}}}{\rho}=\left(\nu \frac{\partial \bar{u}}{\partial y}\right)_{\text {wall }}=v_{b c} \frac{\bar{u}}{y}
$$

where $\tau_{\text {wall }}=u_{\tau}^{2}$ is the wall shear stress. Thus, $v_{b c}$ is the artificial viscosity, which can be found from

$$
v_{b c}=\frac{\bar{u}_{\tau}^{2} y}{\bar{u}}=\frac{\bar{u}_{\tau} y}{\bar{u}^{+}}
$$

where $\bar{u}^{+}$is obtained from Eq. (16), and $v_{b c}$ was used instead of $v$ for the viscosity of the first grid away from the wall boundary. In this way, the instantaneous wall shear stress is used and logarithmic law is used.

Table 2 shows the minimum resolution needed for two different Reynolds numbers. The finest multigrid level achievable was level 5, in which the corresponding nodes are 4,006,240 and the element number is 3,895,296. Comparing level 4 and level 5, the level 4 multigrid level satisfies the resolution requirement listed in Table 2 and gives similar results to level 5. Level 4 was used for most test cases, therefore, the number of nodes was only 514,800 and the number of elements was 486,912 .

Because spatiotemporal resolution of the wake is not required here, attention is focused on the boundary layer of the airfoil and the wake region is not specifically refined.

In this work, for $R e_{c}=150,000$, in the boundary layer $(y / c<$ $0.08)$, a fine mesh is used. The maximal $\Delta x^{+}=53$ and all $\Delta y^{+}$is fixed to 15 . The $\Delta z^{+}$is fixed to 300 , which is the upper limit suggested by Chasnov and Piomelli [13]. This spanwise resolution does not capture the small structure in this direction. As observed [3], the flow is almost 2-D along the midspan and this was assumed to be true for this work. Outside of the boundary layer around the airfoil, all the maximal $\Delta x^{+}, \Delta y^{+}$, and $\Delta z^{+}$are fixed to 300 . This resolution is used for multigrid level 4 .

At the start of simulation, zero initial condition is used for very small Reynolds number. While iterating in time, the Reynolds number increases gradually until the target Reynolds number is reached. The simulation is then allowed to advance until a statistically steady vortex shedding develops. Finally, the simulation is run an additional six shedding cycles to allow calculation of the flow statistics. The time step throughout the simulation is fixed at $10^{-5}$ The experimental results [3] suggest that changing the Reynolds number can significantly affect the boundary-layer development. There exist two distinct flow regimes: 1) boundary-layer separation without reattachment for $R e=100,000$ and 2) flow in the presence of the separation bubble on the upper surface of the airfoil for $R e_{c}=$ 150,000 but reattaches near the trailing edge.

In case 1, experimental results showed that the boundary layer on the upper surface of the airfoil separated at approximately $x / c=$ $0.25(X=-0.225)$. On the other hand, for case $2, R e_{c}=150,000$, the separated shear layer reattaches upstream of $x / c=0.75$ $(X=-0.075)$ and remains attached at the trailing edge. The streamlines of velocity field given in Fig. 2 shows that the LES code captures the important features of the boundary layer including separation, recirculation zone and turbulent boundary layer for flow over the NACA0025 airfoil. Figure 2a shows the flowfield at Reynolds number $100 \times 10^{3}$ and $\alpha=\overline{5^{\circ}}$; the upper surface of the airfoil separates at approximately $x / c=0.35(X=-0.195)$ and did not attach at the trailing edge. Figure $2 \mathrm{~b}$ shows the flowfield at Reynolds number $150 \times 10^{3}$ and $\alpha=5^{\circ}$; the upper surface of the airfoil reattached at approximately $x / c=0.8(X=-0.06)$. The two points A and B indicate a two-vortex boundary-layer bubble. These numerical results agree quite well with experimental observations as it is able to pick up the separation location within $0.1 x / c$. The same flow was numerically investigated [14] with two different numerical codes. First, LES-FLUENT was used on a mesh of 18 million cells (i.e., 90 million degrees of freedom), but was unable to compute boundary-layer separation. Second, an in-house finite-volume-based
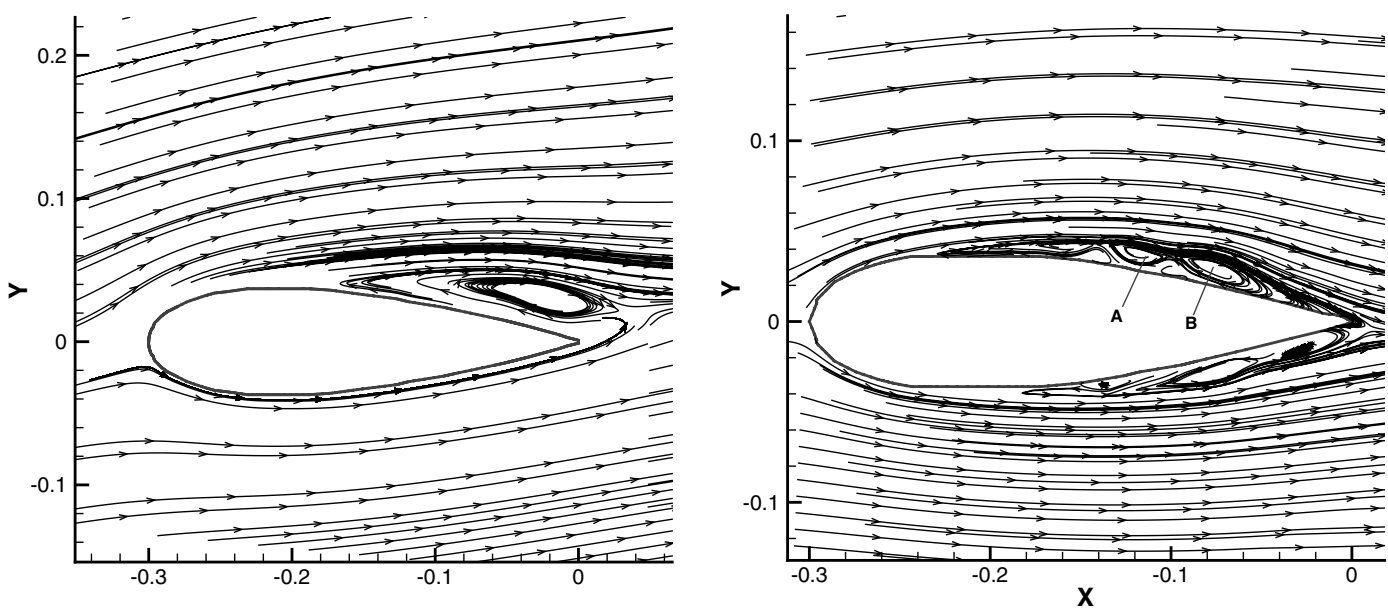

a)

b)

Fig. 2 LES results for instantaneous streamlines of velocity field: a) at Reynolds number $100 \times 10^{3}$ and $\alpha=5^{\circ}$, b) at Reynolds number $150 \times 10^{3}$ and $\alpha=5^{\circ}$. 

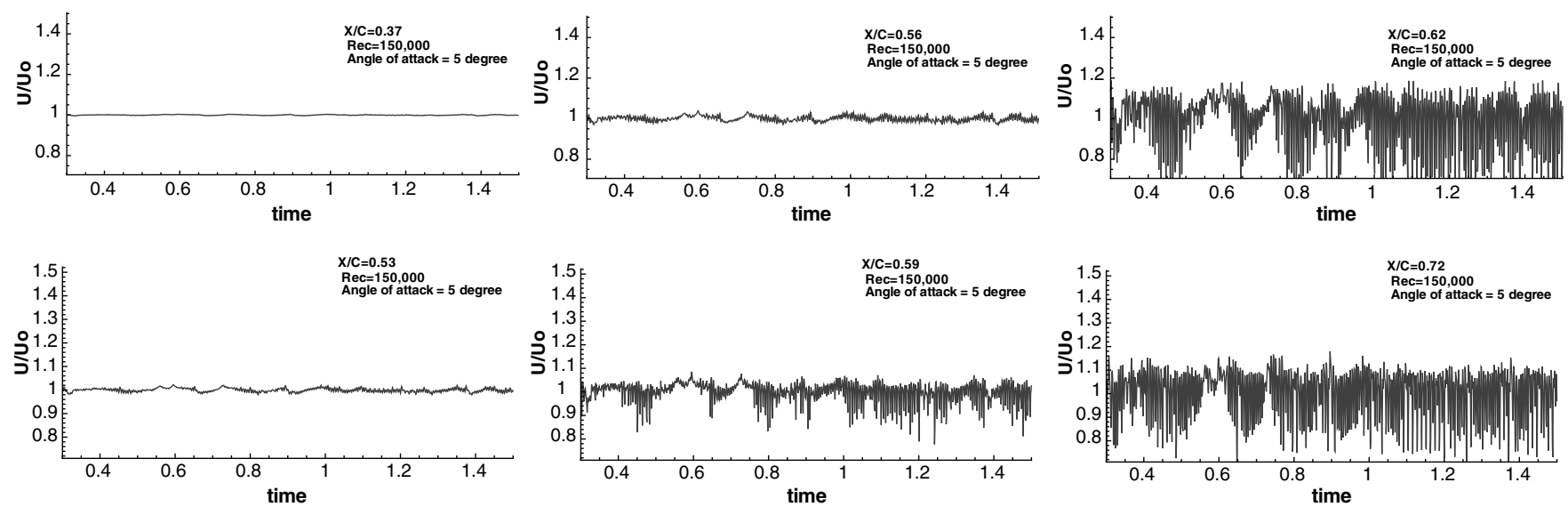

b)

c)

Fig. 3 LES results for streamwise fluctuating velocity component signals at Reynolds number $150 \times 10^{3}$ and $\alpha=5^{\circ}:$ a) at $x / c=0.37(X=-0.189)$ and $0.53(X=-0.141)$, b) $x / c=0.56(X=-0.132)$ and $0.59(X=-0.123)$, and $c) x / c=0.62(X=-0.114)$ and $0.72(X=-0.084)$.

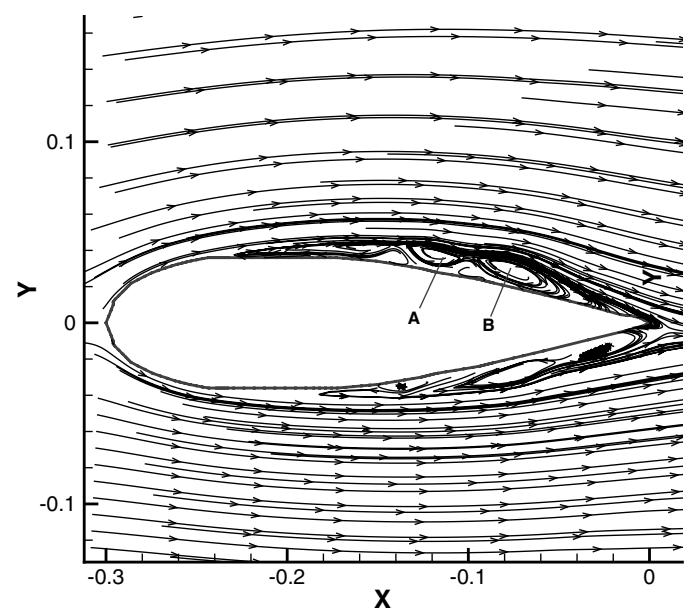

a)

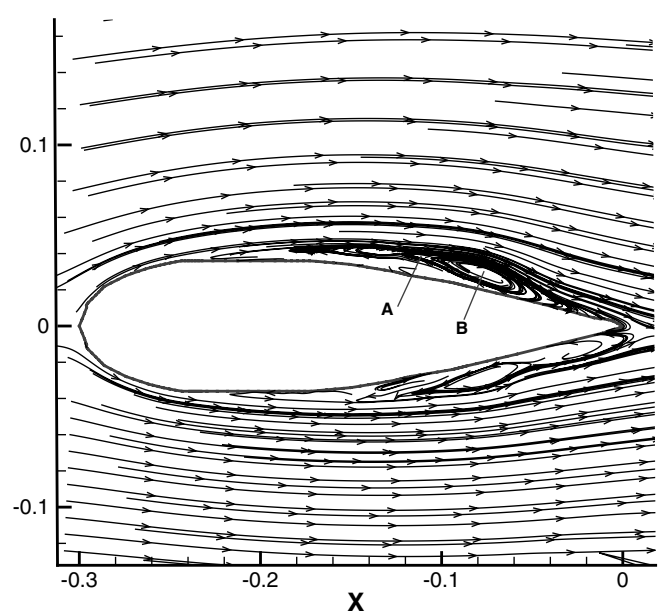

b)

Fig. 4 LES results: vortices merger at Reynolds number $150 \times 10^{3}$, a) at time $=1.100 \mathrm{~s}$ and b) at time $=1.105 \mathrm{~s}$.

code needed 15 million degrees of freedom to capture these flow features. Recall that the current code only requires 2 million degrees of freedom to obtain these results.

Figure 3 reports the evolution of streamwise velocity $u$ during the transition process for $R e_{c}=150,000$ at $\alpha=5^{\circ}$. Initially, no significant fluctuations are observed in the laminar flow region at $x / c=0.37(X=-0.189)$. At $x / c=0.53(X=-0.141)$, a distinct periodic signal appeared. Beyond $x / c=0.62(X=-0.114)$, randomly fluctuating velocity signals were generated instead of periodic velocity fluctuations, which indicates the flow transition to turbulence. The same phenomena was found experimentally [3] and attributed to the growth of the disturbance amplitude to

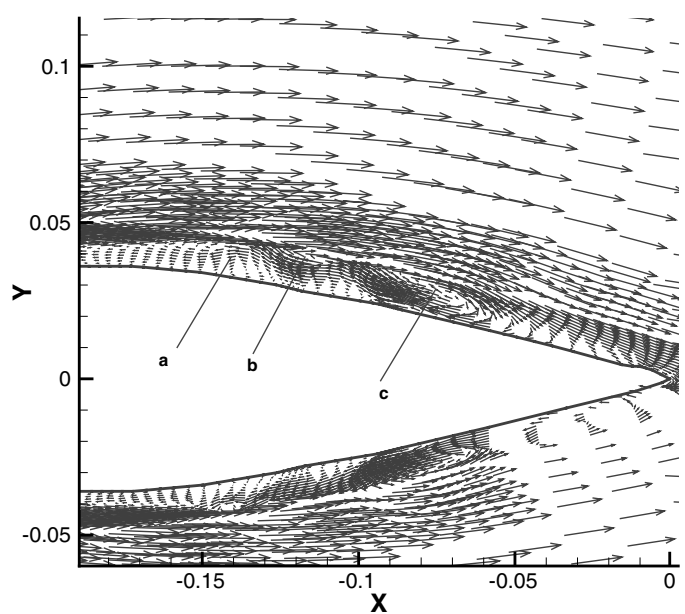

a)

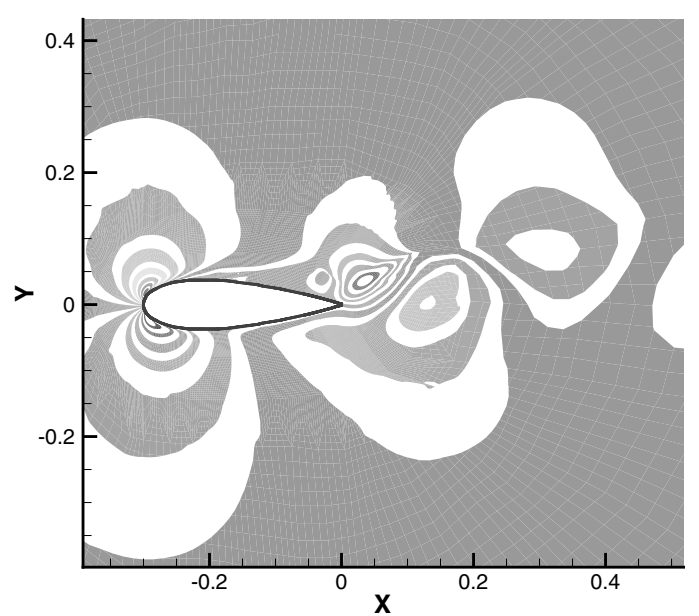

b)

Fig. 5 LES results at Reynolds number $100 \times 10^{3}$ : a) velocity vectors in the boundary layer and b) vortex shedding in the wake. 


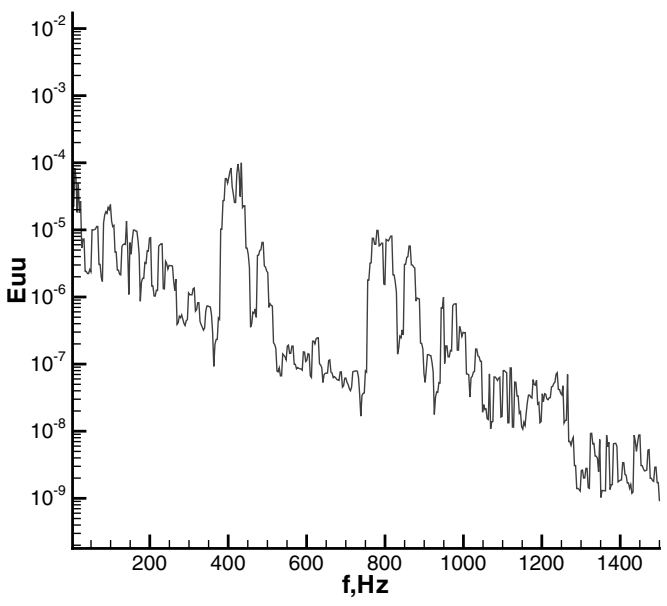

Fig. 6 LES results at Reynolds number $150 \times 10^{3}$ and $\alpha=5^{\circ}$. Spectra of streamwise fluctuating velocity.

Kelvin-Helmholtz instability. This phenomena indicates the location of transition.

The behavior of roll-up vortices is thought to be important in the laminar-to-turbulent flow transition. Figure 4 shows the LES code captures the merger of the two vortices. Vortices marked by $\mathbf{A}$ and $\mathbf{B}$ are shown in Fig. 4a at time $t=1.100 \mathrm{~s}$, but at time $t=1.105 \mathrm{~s}$, in Fig. $4 b$, these two vortices merged. It is speculated [3] that this process results from secondary flow instability before transition.

A detailed investigation of boundary-layer development on the upper surface of the airfoil is performed for $R e_{c}=100,000$ and $R e_{c}=150,000$ at two angles of attack of $0^{\circ}$ and $5^{\circ}$ and compared to experiments [3]. For $R e_{c}=150,000$ at $\alpha=0^{\circ}$, the separation bubble on the upper surface of the airfoil is located between $x / c=0.43$ $(X=-0.171)$ and $0.6(X=-0.12)$ based on the experimental results. The LES predicts the location between $x / c=0.43(X=$ $-0.171)$ and $0.7(X=-0.09)$. Figure 5 a shows the velocity vectors in the boundary layer. The three vortex structures are identified as $a, b$, and $c$. These vortices were observed experimentally [3] and a very clear reattachment occurred after the vortex $a$.

As the Reynolds number decreases to 100,000 , as seen experimentally, significant changes in the boundary-layer development and characteristics occur. The boundary layer on the upper surface separates and fails to reattach, which leads to the formation of a wide wake and significant deterioration of airfoil performance. The boundary layer separates near $x / c=0.35(X=-0.195)$ at all $\alpha$ considered.

Figure 6 shows the spectrum for $R e_{c}=150,000, \alpha=5^{\circ}$, and $x / c=0.3 \overline{5}(X=-0.195)$. Experimentally, the band of the amplified disturbances in the separated shear layer is centered at a high value of $f_{0}=455 \mathrm{~Hz}$ with a second peak reported at approximate $900 \mathrm{~Hz}$. In addition, harmonics are generated, which indicate the onset of transition. The uncertainty of the spectral analysis is approximately $4.5 \%$. A similar transition mechanism can be found in Fig. 6. The band of the amplified disturbances is predicted to be centered at a high value of roughly $f_{0}=420 \mathrm{~Hz}$ and a second peak at approximately $800 \mathrm{~Hz}$. The two main peaks were observed experimentally, which indicates that the LES code captures well the feature of the amplified disturbances.

\section{Conclusions}

A three-dimensional, finite element, time-dependent large-eddy simulation code has been developed to study incompressible turbulent flow over vertical-axis wind turbine blades. The LES code developed is computationally very efficient by using fewer grid nodes, which are capable of capturing boundary-layer bubbles. It is validated for flow over a NACA0025 airfoil. Coherent structures and the laminar bubble separation at different locations downstream of the edge are identified in both the experimental and numerical work. This code is presently being extended to simulate dynamic stall.

\section{Acknowledgment}

This work was supported in part by the Natural Sciences and Engineering Research Council of Canada (NSERC).

\section{References}

[1] Paraschivoiu, I., Wind Turbine Design: With Emphasis on the Darrieus Concept, Presses Internationales Polytechniques, Montreal, Quebec, Canada, 2002

[2] Carmichael, B. H., "Low Reynolds Number Airfoil Survey," NASA 165803,1981

[3] Yarusevych, S., Sullivan, P. E., and Kawall, J. G., "Coherent Structures in an Airfoil Boundary Layer and Wake at Low Reynolds Numbers," Physics of Fluids, Vol. 18, No. 4, 2006, Paper 044101 doi:10.1063/1.2187069

[4] Fasel, H. F., Seidel, J., and Wernz, S., "A Methodology for Simulations of Complex Turbulent Flows," Journal of Fluids Engineering, Vol. 124, No. 4, 2002, p. 933.

doi: $10.1115 / 1.1517569$

[5] Fasel, H. F., von Terzi, D. A., and Sandberg, R. D., "A Methodology for Simulating Compressible Turbulent Flows," Journal of Applied Mechanics, Vol. 73, No. 3, 2006, p. 405. doi:10.1115/1.2150231

[6] Dahlström, S., and Davidson, L., "Large Eddy Simulation of the Flow Around an Aerospatiale A-Airfoil," Proceedings of the European Congress on Computational Methods in Applied Sciences and Engineering, European Community in Computational Methods in Applied Science, Barcelona, Spain, Sept. 2000

[7] Guermond, J. L., and Shen, J., "A New Class of Truly Consistent Splitting Schemes for Incompressible Flows," Journal of Computational Physics, Vol. 192, No. 1, 2003, pp. 262-276. doi:10.1016/j.jcp.2003.07.009

[8] Rannacher, R., and Turek, S., "Simple Nonconforming Quadrilateral Stokes Element," Numerical Methods for Partial Differential Equations, Vol. 8, No. 2, 1992, pp. 97-111. doi:10.1002/num.1690080202

[9] Mullen, J. S., "Development of a Parallel Spectral Element Based Large Eddy Simulation Model for the Flow of Incompressible Fluids in Complex Geometries," Ph.D. Thesis, Brown Univ., Providence, RI, May 1999.

[10] Tobiska, R., "Full and Weighted Upwind Finite Element Methods," Splines in Numerical Analysis, Mathematical Research, edited by J. W. Schmidt and H. Spath, Vol. 52, Akademie Verlag, Berlin, 1989.

[11] Xu, T., "A Numerical Investigation of Airfoil Boundary Layer Using Large Eddy Simulations," Ph.D. Thesis, Univ. of Toronto, Toronto, 2008.

[12] Krajnovic, S., and Davidson, L., "Large-Eddy Simulation of the Flow Around a Ground Vehicle Body," Chalmers Univ. of Technology, Göteborg, Sweden, 2001.

[13] Chasnov, J., and Piomelli, U., "Large-Eddy Simulations: Theory and Applications," Turbulence and Transition Modelling, edited by A. M. Hallback, D. S. Henningson, and P. H. Alfredsson Kluwer Academic, Norwell, MA, 1996, pp. 269-336.

[14] Tajallipour, N., Owlam, B. B., and Paraschivoiu, M., "Self-Adaptive Upwinding for Large Eddy Simulation of Turbulent Flows on Unstructured Elements," Journal of Aircraft, Vol. 46, No. 3, 2009, pp. 915-926. 